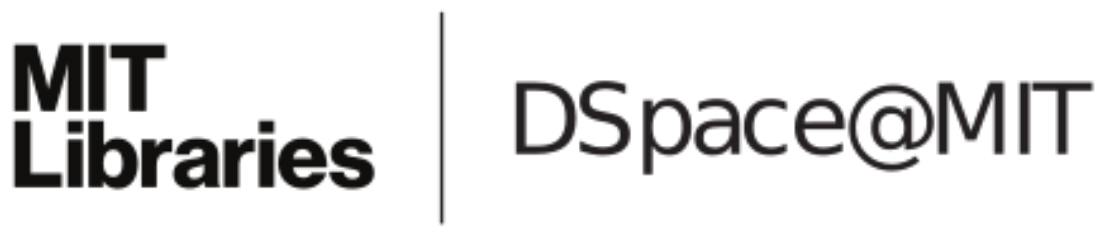

\author{
MIT Open Access Articles
}

\section{Personality influences temporal discounting preferences: Behavioral and brain evidence}

The MIT Faculty has made this article openly available. Please share how this access benefits you. Your story matters.

Citation: Manning, Joshua, Trey Hedden, Nina Wickens, Susan Whitfield-Gabrieli, Drazen Prelec, and John D.E. Gabrieli. "Personality Influences Temporal Discounting Preferences: Behavioral and Brain Evidence." Neurolmage 98 (September 2014): 42-49.

As Published: http://dx.doi.org/10.1016/j.neuroimage.2014.04.066

Publisher: Elsevier

Persistent URL: http://hdl.handle.net/1721.1/99123

Version: Author's final manuscript: final author's manuscript post peer review, without publisher's formatting or copy editing

Terms of use: Creative Commons Attribution 


\title{
Personality Influences Temporal Discounting Preferences: Behavioral and Brain Evidence
}

\author{
Joshua Manning ${ }^{1,2}$, Trey Hedden ${ }^{3,4}$, Nina Wickens ${ }^{1,2}$, Susan Whitfield-Gabrieli ${ }^{1,2}$, Drazen \\ Prelec $^{1,5,6}$, and John D. E. Gabrieli ${ }^{1,2}$ \\ ${ }^{1}$ Department of Brain and Cognitive Sciences, Massachusetts Institute of Technology, 77 \\ Massachusetts Ave., Cambridge, MA 02139 \\ 2McGovern Institute for Brain Research, Massachusetts Institute of Technology, 77 \\ Massachusetts Ave., Cambridge, MA 02139 \\ ${ }^{3}$ Athinoula A. Martinos Center for Biomedical Imaging, Department of Radiology, Massachusetts \\ General Hospital, $14913^{\text {th }}$ St., Suite 2301, Charlestown, MA 02129 \\ ${ }^{4}$ Department of Radiology, Harvard Medical School. 25 Shattuck St., Boston, MA 02115 \\ ${ }^{5}$ Sloan School of Management, Massachusetts Institute of Technology, 77 Massachusetts Ave., \\ Cambridge, MA 02139 \\ ${ }^{6}$ Department of Economics, Massachusetts Institute of Technology, 77 Massachusetts Ave., \\ Cambridge, MA 02139
}

\section{Abstract}

Personality traits are stable predictors of many life outcomes that are associated with important decisions that involve tradeoffs over time. Therefore, a fundamental question is how tradeoffs over time vary from person to person in relation to stable personality traits. We investigated the influence of personality, as measured by the Five-Factor Model, on time preferences and on neural activity engaged by intertemporal choice. During functional magnetic resonance imaging (fMRI), participants made choices between smaller-sooner and larger-later monetary rewards. For each participant, we estimated a constant-sensitivity discount function that dissociates impatience (devaluation of future consequences) from time sensitivity (consistency with rational, exponential discounting). Overall, higher neuroticism was associated with a relatively greater preference for immediate rewards and higher conscientiousness with a relatively greater preference for delayed rewards. Specifically, higher conscientiousness correlated positively with lower short-term impatience and more exponential time preferences, whereas higher neuroticism (lower emotional stability) correlated positively with higher short-term impatience and less exponential time preferences. Cognitive-control and reward brain regions were more activated when higher

(C) 2014 Elsevier Inc. All rights reserved.

Corresponding Author: Joshua Manning, Address: 43 Vassar St., 46-4033A, Cambridge, MA 02139, joshuam@mit.edu, Telephone: 412-951-0815.

Publisher's Disclaimer: This is a PDF file of an unedited manuscript that has been accepted for publication. As a service to our customers we are providing this early version of the manuscript. The manuscript will undergo copyediting, typesetting, and review of the resulting proof before it is published in its final citable form. Please note that during the production process errors may be discovered which could affect the content, and all legal disclaimers that apply to the journal pertain. 
conscientiousness participants selected a smaller-sooner reward and, conversely, when higher neuroticism participants selected a larger-later reward. Both cases involved choices that went against predispositions implied by personality. These findings reveal that stable personality traits fundamentally influence how rewards are chosen over time.

\section{Keywords}

Personality; Decision Making; Delayed Discounting; Reward; fMRI

\section{Introduction}

Many decisions involve a conflict between immediate rewards and delayed gratification between, for example, receiving a smaller cash amount now and a larger cash amount next year. Formal temporal discounting models specify how subjective present value, which is the personal value (utility) of money or a particular good obtained at a specific date, decreases as that date moves further into the future (Frederick et al., 2002). A fundamental question is how such value varies from person to person in relation to stable personality traits. Further, is personality associated with the neurobiological competition between immediate and delayed rewards as decisions are made, or with integration among neural systems involved with both immediate or delayed rewards, dependent on the behavioral predisposition associated with personality type?

Both temporal discounting and the influence of personality traits on behavior have been studied extensively, but there is limited evidence about either how major personality factors, such as the Big Five Personality traits (Costa \& McCrae, 1992), influence temporal discounting or how personality traits and temporal discounting are related with one another in terms of brain processes. Personality traits predict variation in goal-directed behaviors that involve tradeoffs between immediate and delayed consequences, including health and exercise (Conner \& Abraham, 2001), academic performance (Conner \& Abraham, 2001; Paunonen, 2003), years of education (Goldberg et al., 1998), and job performance (Barrick et al., 2001). Each of these endeavors requires sacrifice of current satisfactions in exchange for remote rewards, so it might be hypothesized that personality traits influence temporal discounting.

The most widely used and validated self-report of personality is the Big Five questionnaire (Costa \& McCrae, 1992), which yields independent measures of conscientiousness, neuroticism, extraversion, openness to experience, and agreeableness. One study found that greater extraversion was associated higher discounting rates, but only in people with lower cognitive scores, and that greater neuroticism was associated with higher discounting rates, but only in people with higher cognitive scores (Hirsh et al., 2008). Also, tryptophan depletion increases discounting rates in individuals with higher neuroticism (Demoto et al., 2012). Impulsiveness can also be measured as a trait by a questionnaire. Greater trait impulsivity correlated with preference for immediate rewards and increased discounting rates (Sripada et al., 2012) and also difficulty resisting immediate rewards (Diekhof et al., 2012). Thus, personality variables have been related to variation in discounting rates, but 
there has been no straightforward relation reported between the Big Five personality factors and variation in temporal discounting.

Personality has been linked to the consistency of intertemporal decisions, as revealed by impulsivity and procrastination. Individuals who score high on neuroticism report more impulsive behavior (Whiteside \& Lynam, 2001) and more procrastination (Lee et al., 2006); conversely, conscientiousness is associated with less procrastination (Lee et al., 2006). We therefore hypothesized that greater conscientiousness would be associated with greater willingness to wait for reward, whereas greater neuroticism (or instability that can be associated with impulsiveness) would be associated with lesser willingness to wait for reward.

If personality traits alter temporal discounting preferences, there are two major alternative ways in which this could occur in the brain. Some neuroimaging studies have found that different brain regions are associated with immediate versus delayed rewards (e.g., McClure et al., 2004). These findings raise the possibility that variation in personality may be understood in the framework of a dual-system competition model, in which a fast, visceral system responds to immediate rewards, and a slow deliberate system considers delayed rewards. Personality could tip the balance of this competition. Other neuroimaging studies have found that brain regions appear to integrate information about immediate versus delayed rewards (e.g., Kable \& Glimcher, 2007). These findings raise the alternative possibility that variation in personality may be understood in a systems integration model, in which personality does not influence one or another competing system, but rather multiple systems operating in concert.

Neuroimaging studies of temporal discounting have provided evidence for both competition and integration under varying experimental designs (reviewed in Peters \& Büchel, 2001). Evidence for competition comes from functional neuroimaging that has associated brain responses to immediate rewards with subcortical reward systems, and brain responses to delayed rewards with prefrontal and other neocortical regions thought to support cognitive control (McClure et al., 2004). Regions in the reward system, including the ventral striatum, medial prefrontal cortex, and posterior cingulate cortex, exhibited higher levels of activation when people chose immediate rewards over delayed rewards (McClure et al., 2004). Conversely, regions associated with cognitive control, such as the dorsolateral prefrontal cortex, were associated with the value of the delayed rewards (McClure et al., 2004). The lateral prefrontal cortex also has a causal role in the self-control needed to select delayed rewards (Figner et al., 2010). Evidence for integration comes from the finding that activation in ventral striatum appears to track the subjective value of rewards, calculated as a hyperbolic discount function, simultaneously with regions in prefrontal and posterior cingulate cortices (Kable \& Glimcher, 2007). Regions including the dorsolateral prefrontal cortex, ventrolateral prefrontal cortex, middle temporal gyrus, somatosensory cortex, primary motor cortex, and the striatum were also associated with the subjective value of rewards (Onoda et al., 2011).

Individual differences in reward processing have been associated with individual differences in the magnitude of activation occurring in these neural systems. Increased impulsivity, 
measured by temporal discount functions, was associated with lower activation in the ventral striatum and nucleus accumbens while responding to delayed rewards (Ballard \& Knutson, 2009; Ripke et al., 2012). However, the ventral striatum has also been shown to have higher activation for steeper, more impulsive temporal discounters while waiting for the receipt of a reward (Jimura et al., 2013). Cognitive control regions, including the dorsolateral prefrontal cortex, showed greater deactivation to the delay of reward in impulsive temporal discounters (Ballard \& Knutson, 2009). Thus both reward and cognitive control systems have exhibited variation in activation that was associated with variation in temporal discounting preferences between immediate and delayed rewards.

We employed a single model of constant sensitivity function (Ebert \& Prelec, 2007; Bleichrodt et al., 2009) to examine the relation of personality to both behavior and brain function. We chose this model, relative to other models of temporal discounting, because it offers two formal measures of time preference, one of impatience (pure discounting) and one of impulsivity. The impatience measure captures how much weight people give to future outcomes. The impulsivity measure captures whether time discounting promotes inconsistent decisions about future outcomes, such that, for example, a person's 'morning preferences' might yield a decision to work rather than party in the evening, but his 'evening preferences' would yield the opposite. Using this model, with the separate impulsivity parameter, is especially appropriate because of the established relationship between impulsivity and neuroticism (Whiteside \& Lynam, 2001). We examined whether each of these two temporal-discounting measures correlated with personality-related psychological variables and neural activity.

We therefore aimed to integrate economic, psychological, and neurobiological perspectives to understand how personality differences are associated with making economic choices over time. We characterized the personalities of healthy young adults who performed temporal discounting choices while undergoing functional magnetic resonance imaging (fMRI), and analyzed their time discounting functions. We also measured cognitive abilities because of evidence that such abilities can influence temporal discounting (Shamosh \& Gray, 2008; Shamosh et al., 2008) and interact with the personality trait of extraversion (Hirsh et al., 2008). We hypothesized that conscientiousness would be associated with relatively more consistent time preferences and shallower discounting or preferences for relatively longer delays, and, conversely, that neuroticism would be associated relatively less consistent time preferences and with deeper discounting or preferences for relatively shorter delays.

Functional neuroimaging analyses, analogous to the behavioral analyses, focused on the relation between personality traits and selection of shorter versus longer delays in the context of the amount of subjective value gained by a person's choice. With this type of fMRI analysis, we asked whether personality influences in relation to subjective value would manifest in the brain as variation in competition between subcortical and cortical systems (with personality factors differentially associated with the two systems), or as an integration between subcortical and cortical systems (with personality factors similarly associated with the two systems). 


\section{Method}

\subsection{Participants}

Participants were 40 healthy young adults between ages 20 and 32 (right handed, mean age $=24.9$ years, 22 females) screened for prior neurological disorder. Three participants were excluded from analysis due to invalid data: One had missing fMRI data; one switched hand response button boxes; one had excessive movement that required the removal of 253 outlier data points (threshold of $\mathrm{z}=3$ relative to the mean intensity and composite motion with threshold of $1 \mathrm{~mm}$ relative to previous time point). The study was approved by the Committee On the Use of Humans as Experimental Subjects at MIT in accordance with World Medical Association Declaration of Helsinki. All participants gave informed consent.

\subsection{Procedure}

\subsubsection{Participant Characterization: Personality, Intelligence, and Working} Memory-Participants completed the 60-item Neuroticism-Extraversion-Openness Five Factor Inventory (Costa \& McCrae, 1992). Responses were scored for neuroticism, extraversion, openness to experience, conscientiousness, and agreeableness. Intelligence for each participant was assessed with the Full-Scale Intelligence Quotient (FSIQ) estimate from the American National Adult Reading Test (Wechsler, 1981). Working memory ability was assessed with two tests: (1) the Letter-Number Sequencing test from the WAIS-R (Wechsler, 1981), and (2) a Multiple-category N-back task adapted from Salthouse, Atkinson, \& Berish (2003).

\subsubsection{Neuroimaging}

Behavioral Assessment of Temporal Discounting-During scanning, participants were presented with 108 trials consisting of two options: (1) a smaller monetary reward with a shorter delay and (2) a larger monetary reward with a longer delay. Participants were informed that one of their choices would be randomly selected as a true payoff. One option was presented initially for 2-8 seconds (counterbalanced across shorter and longer delays), followed by both options presented until the participant made a choice or the trial timed out (after a total of 14 seconds). A fixation cross was presented for the remaining time after the choice. Options were presented side-by-side, and the order and side of shorter and longer delays was fully counterbalanced across trials (Figure 1).

Sixty trials contained delays of 0 (immediate), 21, 60, 180, and 365 days, with rewards ranging from $\$ 30$ to $\$ 150$. These intervals were selected to approximate $\log ($ time) intervals. The remaining 48 of the 108 trials were adaptive. This adaptive method assured that some trials provided options that were close to equivalent in subjective value. Using the starting values from the practice session increased the likelihood that the in-scanner adaptive trials more rapidly converged toward each participant's indifference point. The model was fit to all trials in the scanner, both fixed and adaptive options. This design enhanced the accurate estimation of the discounting model by increasing the number of observations most sensitive to a participant's subjective value in decision making. One of the options was always an immediate reward and the other was a delayed reward of $\$ 60$ and $\$ 150$ at each of the four delays. The immediate alternatives for each reward/delay pair were determined in an 
adaptive fashion using a staircase procedure. The starting value of the immediate alternative for each reward/delay pair was determined from the participant's behavior during a pre-scan session (described below). Depending upon the participant's responses to these trials, the next immediate reward was either raised or lowered, with the increments of change becoming successively smaller in log amounts. The minimum reward for adaptive trials varied across participants dependent on the adaptive reward values from the staircase procedure. After scanning, participants received the reward from their choice on a randomly selected trial as a check. If there was a delay for the selected choice, a check was mailed to the participants at the scheduled date.

Prior to the fMRI session participants received 96 practice adaptive trials outside of the scanner with the same delays used in the MRI scanner. Starting values of $\$ 60$ and $\$ 150$ were paired with the delays and were adapted using the procedure above. The first staircase trial for each delayed reward was paired with an immediate reward alternative that was $1 / 2$ of the log-delayed amount (rounded to the nearest dollar). Depending upon the participant's responses to these trials, the next immediate reward was either raised or lowered, with the increments of change becoming successively smaller in log amounts. The final values from this adaptive procedure were used as starting values for the adaptive trials during scanning.

2.2.3. Discounting Analysis-Data from the discounting task in the scanner were used to model each participant's subjective value using the constant sensitivity discounting function (Ebert \& Prelec, 2007; Bleichrodt et al., 2009), (Eq. 1), which allows formal separation of impatience levels and impulsivity and relate them to conscientiousness and neuroticism. In this model, $\beta$ is a measure of pure exponential discounting or impatience. As $\beta$ increases, people become more impatient. Time-sensitivity is measured by $a$. Rational compound discounting is equivalent to $a=1$. As $a$ decreases, people become increasingly insensitive to differences between future time points. The limiting case $a \sim 0$ yields dichotomous discounting, where an individual only distinguishes between 'now' and 'later,' treating all future dates as equivalent. This would promote extreme inconsistency in intertemporal choices. In principle, inter-subject variation in $\alpha$ and $\beta$ across people describes individual differences in temporal discounting. Maximum likelihood estimation, with the softmax activation function (Eq. 2) for the likelihood, was used to obtain the parameter estimates $a$ and $\beta$ from equation 1, and $\theta$, the inverse temperature or slope parameter from the softmax function. $\theta$ reflects a random element in choice. $\theta=0$ represents a person that choses completely at random. As $\theta$ increases a person is more likely to choose the option with the highest subjective value. These parameters were estimated for each participant, and we related these discounting functions to each individual's personality measures. We calculated both Pearson correlations and Spearman rank correlations between each participant's estimated discounting parameters and personality measures. Because previous research has found a correlation between personality and both IQ and working memory (Shamosh \& Gray, 2008; Shamosh et al., 2008), we also examined the Pearson correlations between personality and both IQ and working memory.

$$
f(t)=\exp \left(-(\beta t)^{\alpha}\right) \quad \text { Equation } 1
$$




$$
\mathrm{p}\left(\text { Option Chosen }_{i}\right)=\frac{e^{\theta \times f\left(t_{i}\right) * \text { Reward Chosen }}}{e^{\theta \times f\left(t_{i}\right) * \text { Reward Chosen }}+e^{\theta \times f\left(t_{j}\right) * \text { Reward Rejected }}} \quad \text { Equation } 2
$$

2.2.4. fMRI Acquisition-Data were acquired using a 3-Tesla Siemens Tim Trio scanner (Siemens, Erlangen, Germany) with a 12-channel phased array whole-head coil. 3D T1weighted magnetization prepared rapid acquisition gradient echo (MP-RAGE) anatomical images $\left(\mathrm{TR}=2530 \mathrm{~ms}, \mathrm{TE}=3.39 \mathrm{~ms}\right.$, flip angle $=7^{\circ}, 1.33 \mathrm{~mm}$ slice thickness, $1.3 \times 1 \mathrm{~mm}^{2}$ in plane resolution) and $\mathrm{T} 2 *$ weighted EPI sequence functional images $(\mathrm{TR}=2.0 \mathrm{sec}, \mathrm{TE}=$ $30 \mathrm{~ms}$, flip angle $=90^{\circ}, 3 \mathrm{~mm}^{3}$ resolution, 300 timepoints per run with 3 runs, tilt $=22^{\circ}$ upward from AC-PC line to minimize distortion and signal dropout, interleaved acquisition, using prospective acquisition correction) (Thesen, et al., 2000) with full brain coverage were collected.

2.2.5 fMRI Analysis-The functional data were analyzed using SPM8 (http:// www.fil.ion.ucl.ac.uk/spm/software/spm8/, 2013). Functional images were preprocessed with realignment for motion correction, slice-time correction, artifact detection (threshold of $\mathrm{z}=3$ relative to the mean intensity and composite motion with threshold of $1 \mathrm{~mm}$ relative to previous time point), spatial smoothing (6mm full-width-half-maximum Gaussian kernel), and ANTS (Avants, 2009) normalization and coregistration of the contrast images to each participant's anatomical scan using Freesurfer (Fischl et al., 2001; Fischl et al., 2002).

We used a general linear model (GLM) for the fMRI analysis that related personality variables to individually calculated subjective value for each participant. The GLM consisted of three vectors of onsets. The first corresponded to trials where participants chose the option with a shorter delay, and the second corresponded to trials where participants chose the option with a longer delay. Both onset vectors for choice consisted of unit values at the time of the presentation of the second option. A third vector corresponded to the presentation of the first option. Additional parametric regressors coincided with the onsets at presentation of the second option. Nuisance regressors were also included in the GLM; one for each artifactual time point, one for linear drift, and 7 motion parameters ( 3 rotation, 3 translation, and 1 composite). Three sessions were concatenated for the design matrix (See supplementary Figure 1).

These parametric regressors represented the utility surplus of each decision; the difference in the subjective value between the option chosen and the option not chosen (all fMRI contrasts are with the parametric regressors). The utility surplus captures the subjective value of the entire option, which provides more information than other possible regressors (e.g., absolute monetary value or subjective value of a single option). This is also an important measure because we predicted a relationship between personality and the parameters of the constant sensitivity model, which determines each person's unique subjective value.

One parametric regressor corresponded to trials where the option with a shorter delay was chosen and the other regressor corresponded to trials where the option with a longer delay 
was chosen. For chosen shorter delays, the regressor was calculated as the subjective value of the option with the shorter delay minus the subjective value of the option with the longer delay (Utility Surplus Short). For chosen longer delays, the regressor was calculated as the subjective value of the option with the longer delay minus the subjective value of the option with the shorter delay (Utility Surplus Long) (See supplementary Table 1 for distribution of choices). All subjective values were calculated by multiplying Eq. 1 by the monetary value of the options (assuming that utility was linear in money). When positive, this difference of the subjective value shows how much additional utility the participant gained by choosing the option with the larger modeled subjective value (based on their entire series of choices). When negative, this difference shows how much additional utility the participant could have received had they chosen the option with the larger modeled subjective value.

Because we were interested in the relationship between discounting, personality, and brain function, the subjective values associated with each trial were essential to evaluate these relationships, as opposed to other possible variables (e.g. actual values of the option chosen or the difference between the two monetary values). In addition we hypothesized a relationship between neuroticism and high impulsivity/high short-term impatience, and conscientiousness and low impulsivity/low short-term impatience. Therefore we separated these regressors dependent on whether a person chose the shorter delay or the longer delay in order to see how the preference for the length of delay related to different personality traits and brain function. Finally, we used the difference in subjective value or utility surplus because this value represents both the difficulty of the choice and the actual utility gained or lost on each trial.

We used the personality scores of conscientiousness and neuroticism as second level covariates in separate second level group analyses. Two separate second level group analyses avoided collinearity between the personality dimensions. Conjunctions of thresholded (FDR < .05) correlation maps were performed (Nichols et al., 2008). All analyses applied a cluster level false discovery rate (FDR) threshold of $\mathrm{p}<.05$.

\section{Results}

\subsection{Behavioral Results}

We used an intertemporal choice task in which participants chose between two options: (1) a smaller monetary reward with a shorter delay, and (2) a larger monetary reward with a longer delay (Figure 1). Each participant's data was fit to the two-parameter constant sensitivity function (Ebert \& Prelec, 2007; Bleichrodt et al., 2009). One parameter, $a$ (where $0 \leq a \leq 1)$ measures time-sensitivity and impulsivity. Smaller $a$ reflects greater impulsivity (less rational) and more short-term impatience, while $a=1$ reflects a rational discounter. The second parameter, $\beta$ (where $0 \leq \beta$, ) measures exponential discounting or pure impatience. Larger $\beta$ reflects greater overall impatience. The distribution of the timesensitivity/impulsivity parameter $(\alpha)$ was approximately Gaussian except for being truncated at 1 , but the distribution of the pure-discounting parameter $\beta$ was truncated at zero and skewed to the right, resembling an exponential distribution. Therefore, we took the log transform of $\beta$ to make the data more Gaussian. We assessed each participant's personality traits with the 60-item Neuroticism-Extraversion-Openness Five Factor Inventory (Costa \& 
McCrae, 1992). The influence of personality factors on temporal discounting was analyzed by calculating Pearson correlations between personality factors and the discounting parameters $\alpha$ and $\log (\beta)$. Based on our hypotheses, we examined correlations of neuroticism and conscientiousness on an a priori basis, but also performed an exploratory analysis of extraversion, agreeableness, and openness.

As hypothesized, neuroticism was negatively correlated with $a(r=-.41, p=.008)$. Conscientiousness was positively correlated with $a(r=.47, p=.002)$ (Figure 2 and supplementary Table 1 for distribution of personality scores). The other three personality factors did not correlate with $a(p>.13)$. However, our hypotheses regarding $\beta$ were not supported, as there were no correlations between personality and $\log (\beta)(\mathrm{p}>.33)$. The correlation with $a$ indicates that personality was related to impulsivity differences and deviations from exponential discounting toward hyperbolic discounting. The lack of correlation between personality and $\log (\beta)$ indicates personality was not related to exponential discounting when separating impulsivity from the exponential parameter. In addition, conscientiousness was negatively correlated with neuroticism in these participants $(r=-.33, p=.037)$. There were no significant correlations between $a$ and $\log (\beta)$, or between either $\alpha$ or $\log (\beta)$ and variation in cognitive abilities as measured by an IQ estimate or two measures of working memory capacity.

Because $a$ is constrained at 1, it is difficult to assess the normality of $a$. Therefore, we also calculated the Spearman rank correlations between personality factors and discounting parameters $\alpha$ and $\log (\beta)$. The results were highly similar to the Pearson correlations. Neuroticism was negatively correlated with $a(r=-.40, p=.005)$ and conscientiousness was positively correlated with $a(r=.44, p=.005)$. As with the Pearson correlation there were no significant correlations between $\log (\beta)$ and personality traits. There were also no correlations between either $a$ or $\log (\beta)$ and variation in cognitive abilities as measured by an IQ estimate or two measures of working memory capacity. However, $\alpha$ and $\log (\beta)$ were negatively correlated using the Spearman rank correlation $(r-.79, p<.001)$.

We did not find the correlation between $a$ and $\log (\beta)$ using the Pearson correlation that we did with the Spearman correlation, which is likely due to the lack of normality. However, the significant negative Spearman rank correlation between these two parameters is consistent with the expected direction of the relationship. Increasing $a$ is associated with greater shot-term patience and decreasing $\log (\beta)$ is associated with greater patience overall. Therefore, the negative correlation supports the expected relationship between the two parameters. All correlations were calculated in separate models. We also assessed the goodness-of-fit of the constant sensitivity model. The model was fit separately to the 40 participants. The model was a good fit for $85 \%$ of the participants using a $\chi^{2}$ threshold of . 05.

We also asked participants to report annual income in eight income brackets from $\$ 0$ to over $\$ 250,000$. Greater income was marginally correlated with lower $a(r=-.33, p=.055)$. Many participants were young adults in college or graduate school, so there may have been an atypical relation between present and anticipated future earnings. 


\section{2 fMRI Results}

\section{Subjective Value In Relation to Choosing Shorter vs. Longer Delays-We}

examined the relations of neuroticism and conscientiousness to intertemporal choice in the brain because only these personality factors correlated behaviorally with such choices. Because neuroticism and conscientiousness were negatively correlated, two separate second level group analyses were conducted to avoid collinearty between the two personality dimensions. We estimated each participant's utility surplus, the difference in the subjective value between the option chosen and the option not chosen, on each trial. The first contrast examined the utility surplus, relative to each participant's subjective value (SV) of the two options presented, between trials where a shorter delay was chosen versus those where a longer delay was chosen (Utility surplus short vs. Utility surplus long). There were no significant activations correlated with neuroticism. However, greater conscientiousness correlated positively with increased activation in ventral and dorsal striatum, bilateral DLPFC, left OFC, precuneus, and bilateral superior parietal lobe (Figure 3 and Supplementary Table 2).

The second contrast examined utility surplus, relative to each participant's SV of the two options presented, between selecting a longer delay versus a shorter delay (Utility surplus long vs. Utility surplus short). There were no significant activations correlated with conscientiousness, but greater neuroticism correlated positively with greater activations in ventral striatum, bilateral DLPFC, bilateral insula, ACC, MPFC, and bilateral OFC (Figure 4 and Supplementary Table 3).

Next we examined the variation in left and right ventral striatum activations that associated personality and intertemporal choice (Figure 5). Individual parameter values were extracted from the overlap between the anatomically defined head of the caudate, which includes surrounding tissue of the ventral striatum (WFU_PickAtlas, Maldjian et al., 2003) and the larger clusters of correlation between the two personality factors and intertemporal choices that were found in each second-level analyses (i.e., conscientiousness in Figure 3 and neuroticism in Figure 4). Contrast values were then extracted from each individual's firstlevel contrast images. For both the Utility Surplus Short vs. Utility Surplus Long contrast correlated with conscientiousness and the Utility Surplus Long vs. Utility Surplus Short contrast correlated with neuroticism, increasing contrast values from the first level contrast map were positively associated with personality scores.

There were apparent similarities in activations associated with both personality dimensions of conscientiousness and neuroticism, and these apparent co-localizations were statistically examined in a conjunction analysis (Nichols et al., 2008) (FDR corrected threshold of p < . 05). There was indeed substantial overlap in the brain regions associated with conscientiousness or neuroticism (Figure 6). Selecting the option that was behaviorally opposed to a person's discounting tendency (i.e., selecting the shorter delay for a person with high conscientiousness or selecting the longer delay for a person with high neuroticism) was associated with widespread activation of areas associated with reward (ventral striatum) and with cognitive control (DLPFC). 
To be certain that these fMRI results were not the due to working harder or taking longer when choosing the option that was behaviorally opposed to a person's discounting tendency, we analyzed the correlations between neuroticism and response time when the longer delay was chosen and between conscientiousness and response time when the shorter delay was chosen. There were no significant correlations in either case (ps $>.21$ ). High neuroticism scores were not associated with taking longer when choosing the longer delay, nor were high conscientiousness scores associated with taking longer when choosing the shorter delay.

\section{Discussion}

Economists and psychologists have described many differences in people's discounting preferences and behaviors (Frederick et al., 2002), and here we describe a convergence of behavioral economics, personality, and brain function that appears to contribute to such individuality. With respect to personality, higher conscientiousness correlated positively with lower short-term impatience and more exponential time preferences, while higher neuroticism correlated positively with higher short-term impatience and less exponential time preferences. Thus, the estimated discount functions of people with high neuroticism implies strong impatience for short delays, but relatively less impatience when the same trade-off between delay and monetary amount is moved into the future. This discounting profile would promote temporally inconsistent behavior, where a decision made in the morning might be reversed in the evening. In contrast, the discount functions of people with high conscientiousness exhibits both lower impatience with respect to short term delays, as well as more time consistency.

The relation between personality and temporal discounting can be illustrated with examples of differences in subjective values for immediate versus delayed rewards for the participant who had the highest neuroticism score versus the participant who had the highest conscientiousness score. Assume both are offered the option of $\$ 8$ now or $\$ 10$ in five days. Using their implied discount model, for the highly neurotic person, the subjective value of $\$ 10$ in five days was $\$ 4.87$, which was lower than the subjective value of $\$ 8$ now. For the highly conscientious person the subjective value of $\$ 10$ in five days was $\$ 9.93$, which was higher than the subjective value of $\$ 8$ now. Therefore, the highly neurotic person will choose the $\$ 8$ now while the highly conscientious person will choose the $\$ 10$ in five days.

We did not observe significant relations between cognitive measures of a verbal IQ estimate or two complex working memory tasks conceptualized as indexing executive functions. This differs from prior findings that related higher scores on measures of intelligence and working memory capacity with lower temporal discounting (Shamosh \& Gray, 2008; Shamosh et al., 2008) and the interaction of extraversion and emotional stability/reversed neuroticism with cognitive ability and temporal discounting (Hirsh et al., 2008). The lack of convergence with our data may reflect a restricted high range of cognitive abilities in the present study (Mean IQ $=120$ ). In any case, the absence of a relation between cognitive measures and temporal discounting in the present study indicates that influence of personality on temporal discounting preference was not secondary to variation in cognitive ability. 
With respect to brain function, the regions activated have been observed in prior studies of temporal discounting, such as those associated with reward (e.g., striatum) and cognitive control (e.g., prefrontal cortex) (McClure et al., 2004; Kable \& Glimcher, 2007), but the activations showed a novel pattern in relation to personality. Greater activations in brain regions occurred as options were chosen that were contradictory to personality preferences, i.e., when more conscientious people chose the shorter delay or more neurotic people chose the longer delay. Many of these contradictory choices were rational, but it appears that making a choice that contradicts a disposition may require more mental resources that are reflected in greater activations. This is analogous to evidence of greater brain activation when people perform tasks that are inconsistent versus consistent with cultural preferences (Hedden et al., 2008). The present findings may be contrasted with other conditions of temporal discounting in which reward regions respond selectively to immediate rewards whereas regions associated with cognitive control respond similarly to immediate and delayed rewards (McClure et al., 2004). Here, brain regions supporting reward and cognitive control appeared to operate in an integrated fashion for an economic decision that was contradictory to personality preferences.

Prior studies have related individual differences in impulsivity to variation in temporal discounting and in brain function. One study found a relation between individual differences in trait impulsivity, measured by a questionnaire, and activation in MPFC (Sripada et al., 2011). Another study dissociated the subjective valuation stage from the choice stage in such decisions, and found that greater impulsivity, measured by preferences in intertemporal choices, was associated with reduced activations in lateral prefrontal regions at the choice stage (Liu et al., 2012). Other similar cognitive control regions, including the DLPFC, showed a greater decrease in activation for delayed reward in impulsive discounters (Ballard $\&$ Knutson, 2009). Impulsive discounters also showed decreased activation in the ventral striatum while responding to delayed rewards (Ballard \& Knutson, 2009; Ripke et al., 2012), but showed increased activation in the ventral striatum while anticipating the outcome of a delayed reward (Jimura et al., 2013). People with high trait impulsivity showed decreased activation in the nucleus accumbens/ventral striatum when successfully resisting choosing suboptimal immediate rewards (Diekhof et al., 2012). Although the NEO-FFI does not provide a direct measure of impulsivity, neuroticism scores have been associated with high impulsivity (Whiteside \& Lynam, 2001). The present study is thus in accord with these prior neuroimaging studies in identifying the ventral striatum, MPFC, and DLPFC regions as being related to personality-related variation in intertemporal choice.

The present findings occurred in the context of a specific model of temporal discounting, constant sensitivity, and a specific experimental measure of temporal discounting. The constant sensitivity model allowed for separation of pure discounting or impatience, represented by $\beta$, which was not associated with personality variation, from impulsivity/ short-term impatience and preference consistency, represented by $a$, which was associated with variation in both conscientiousness and neuroticism. Other discounting models, such as the simple hyperbolic model, do not separate discounting from impulsivity (both are captured in a single parameter). Another feature of our experimental measure that may be beneficial for estimating discount rates was the adaptive procedure used in some of the 
trials. The adaptive procedure ensured that participants were presented with difficult trials with options near their indifference point, which is important for estimating the model more accurately for each individual.

The present study documented individual differences in economic decision-making and brain function in relation to two fundamental dimensions of human personality, conscientiousness or neuroticism. These personality factors have wide-ranging relations to important human behaviors and outcomes. These two personality factors are predictive of job performance (Hurtz \& Donovan, 2000), and in longitudinal studies higher conscientiousness is a predictor of longevity (Friedman et al., 1993) and lower risk for Alzheimer's disease (Wilson et al., 2007). The present study suggests that these personality factors are associated with quite different valuations of the short-term and the long-term, but that both factors are associated with predispositions that require similar integrated neural resources of reward and cognitive control regions when a specific choice violates that predisposition. Thus, the mental and neural characteristics associated with stable personality traits wield considerable power over the choice between immediate and delayed gratification.

\section{Supplementary Material}

Refer to Web version on PubMed Central for supplementary material.

\section{Acknowledgments}

This work was conducted at the Athinoula A. Martinos Imaging Center at The McGovern Institute for Brain Research, MIT and supported by NIH/NIA R21 AG030770. T.H. is supported by NIH/NIA K01 AG040197. We thank Jack Murtagh and Aaron Mattfeld for assistance with the manuscript.

\section{References}

Avants BB, Tustison N, Song G. Advanced Normalization Tools (ANTS). Penn Image Computing and Science Laboratory. 2009

Ballard K, Knutson B. Dissociable neural representations of future reward magnitude and delay during temporal discounting. Neuroimage. 2009; 45:143-150. [PubMed: 19071223]

Barrick MR, Mount MK, Judge TA. Personality and performance at the beginning of the new millennium: What do we know and where do we go next. Personality and Performance. 2001; 9:930.

Bleichrodt H, Rohde K, Wakker P. Non-hyperbolic time inconsistency. Games and Economic Behavior. 2009; 66:27-38.

Chamorro-Premuzic T, Furnham A. Personality predicts academic performance: Evidence from two longitudinal university samples. Journal of Research in Personality. 2003; 37:319-338.

Conner M, Abraham C. Conscientiousness and the theory of planned behavior: Toward a more complete model of the antecedents of intentions and behavior. Personality and Social Psychology Bulletin. 2001; 27:1547-1561.

Costa, PT.; McCrae, RR. NEO PI-R professional manual: Revised NEO Personality Inventory (NEO PI-R) and NEO Five-Factor Inventory (NEO-FFI). Odessa, FL: Psychological Assessment Resources; 1992.

Demoto Y, Okada G, Okamoto Y, Kunisato Y, Aoyama S, Onoda K, Munakata A, Nomura M, Tanaka SC, Schweighofer N, Doya K, Yamawaki S. Neural and personality correlates of individual differences related to the effects of acute tryptophan depletion on future reward evaluation. Neuropsychobiology. 2012; 65:55-64. [PubMed: 22222380] 
Diekhof EK, Nerenberg L, Falkai P, Dechent P, Baudwig J, Gruber O. Impulsive personality and the ability to resist immediate reward: An fMRI study examining interindividual differences in the neural mechanisms underlying self-control. Human Brain Mapping. 2012; 33:2768-2784. [PubMed: 21938756]

Ebert J, Prelec D. The fragility of time: Time-insensitivity and valuation of near and far future. Management Science. 2007; 53:1423-1438.

Figner B, Knoch D, Johnson E, Krosch A, Lisanby S, Fehr E, Weber E. Lateral prefrontal cortex and self-control in intertemporal choice. Nature Neuroscience. 2010; 13:538-539.

Frederick S, Loewenstein G, O’Donoghue T. Time discounting and time preference: A critical review. Journal of Economic Literature. 2002; 40:351-401.

Friedman H, Tucker J, Tomlinson-Keasey C, Schwartz J, Wingard D, Criqui M. Does childhood personality predict longevity? Journal of Personality and Social Psychology. 1993; 65:176-185. [PubMed: 8355139]

Fischl B, Liu A, Dale AM. Automated manifold surgery: constructing geometrically accurate and topologically correct models of the human cerebral cortex. IEEE Trans Med Imaging. 2001; 20:70-80. [PubMed: 11293693]

Fischl B, Salat DH, Busa E, Albert M, Dieterich M, Haselgrove C, van der Kouwe A, Killiany R, Kennedy D, Klaveness S, Montillo A, Makris N, Rosen B, Dale AM. Whole brain segmentation: automated labeling of neuroanatomical structures in the human brain. Neuron. 2002; 33:341-355. [PubMed: 11832223]

Goldberg JM, Sweeney D, Merenda PF, Hughes JE. Demographic variables and personality: The effects of gender, age, education, and ethnic/racial status on self-descriptions of personality attributes. Personality and Individual Differences. 1998; 24:393-403.

Grober E, Sliwinski M. Dual-task performance in demented and nondemented elderly. Journal of Clinical and Experimental Neuropsychology. 1991; 13:667-676. [PubMed: 1955523]

Hedden T, Ketay S, Aron A, Markus H, Gabrieli JDE. Cultural influences on neural substrates of attentional control. Psychological Science. 2008; 19:12-17. [PubMed: 18181784]

Hirsch JB, Morisano D, Peterson JB. Delay discounting: Interactions between personality and cognitive ability. Journal of Research in Personality. 2008; 42:1646-1650.

Hurtz G, Donovan J. Personality and job performance: The big five revisited. Journal of Applied Psychology. 2000; 85:869-879. [PubMed: 11125652]

Jimura K, Chushak MS, Braver TS. Impulsivity and self-control during intertemporal decision making linked to the neural dynamics of reward value representation. The Journal of Neuroscience. 2013; 33:344-357. [PubMed: 23283347]

Kable J, Glimcher P. The neural correlates of subjective value during intertemporal choice. Nature Neuroscience. 2007; 10:1625-1633.

Lee D, Kelly KR, Edwards JK. A closer look at the relationships among trait procrastination, neuroticism, and conscientiousness. Personality and Individual Differences. 2006; 40:27-37.

Liu L, Feng T, Wang J, Li H. The neural dissociation of subjective valuation from choice processes in intertemporal choice. Behavioural Brain Research. 2012; 231:40-47. [PubMed: 22406016]

McClure S, Laibson D, Loewenstein G, Cohen J. Separate neural systems value immediate and delayed monetary reward. Science. 2004; 306:503-507. [PubMed: 15486304]

Nichols T, Brett M, Andersson J, Wager T, Poline J. Valid conjunction inference with the minimum statistic. Neuroimage. 2005; 25:653-660. [PubMed: 15808966]

Onoda K, Okamoto Y, Kunisat Y, Aoyama S, Shishsida K, Okada G, Tanaka SC, Schweighofer N, Yamaguchi S, Doya K, Yamawaki S. Inter-individual discount factor differences in reward prediction are topographically associated with caudate activation. Experimental Brain Research. 212:593-601. [PubMed: 21695536]

Paunonen S. Big five factors of personality and replicated predictions of behavior. Journal of Personality and Social Psychology. 2003; 84:411-424. [PubMed: 12585813]

Peters J, Büchel C. The neural mechanisms of inter-temporal decision-making: understanding variability. Trends in Cognitive Sciences. 2011; 15:227-239. [PubMed: 21497544] 
Ripke S, Hübner T, Mennigen E, Müller KU, Rodehacke S, Schmidt D, Jacob MJ, Smolka MN. Reward processing and intertemporal decision making in adults and adolescents: The role of impulsivity and decision consistency. Brain Research. 2012; 1478:36-47. [PubMed: 22940231]

Salthouse TE, Atkinson TM, Berish DE. Executive functioning as a potential mediator of age-related decline in normal adults. Journal of Experimental Psychology: General. 2003; 4:566-594. [PubMed: 14640849]

Shamosh N, Gray J. Delay discounting and intelligence: A meta-analysis. Intelligence. 2008; 36:289_ 305.

Shamosh N, DeYoung C, Green A, Reis D, Johnson M, Conway A, Engle R, Braver T, Gray J. Individual differences in delay discounting: Relation to intelligence, working memory, and anterior prefrontal cortex. Psychological Science. 2008; 19:904-911. [PubMed: 18947356]

Sripada C, Gonzalez R, Phan K, Liberzon I. The neural correlates of intertemporal decision-making: Contributions of subjective value, stimulus type, and trait impulsivity. Human Brain Mapping. 2011; 32:1637-1648. [PubMed: 20886577]

Thesen S, Heid O, Mueller E, Schad LR. Prospective acquisition correction for head motion with image-based tracking for real-time fMRI. Magnetic Resonance in Medicine. 2000; 44:457-465. [PubMed: 10975899]

Wechsler, D. WAIS-R Manual: Wechsler Adult Intelligence Scale-Revised. New York, NY: Psychological Corp; 1981.

Wilson R, Schneider J, Arnold S, Bienias J, Bennett D. Conscientiousness and incidence of Alzheimer Disease and mild cognitive impairment. Archives of General Psychology. 2007; 64:1204-1212.

Whiteside SP, Lynam DR. The five factor model and impulsivity: Using a structural model of personality to understand impulsivity. Personality and Individual Differences. 2001; 30:669-689.

http://www.fil.ion.ucl.ac.uk/spm/softwar/spm8/ 


\section{Highlights}

- We model temporal discounting and relate it to personality and fMRI BOLD signal.

- Neuroticism is correlated with greater short-term impatience.

- Higher conscientiousness is correlated with lower short-term impatience.

- Neuroticism correlates with control-reward networks when choosing later rewards.

- Conversely, conscientiousness with control-reward networks for sooner rewards. 


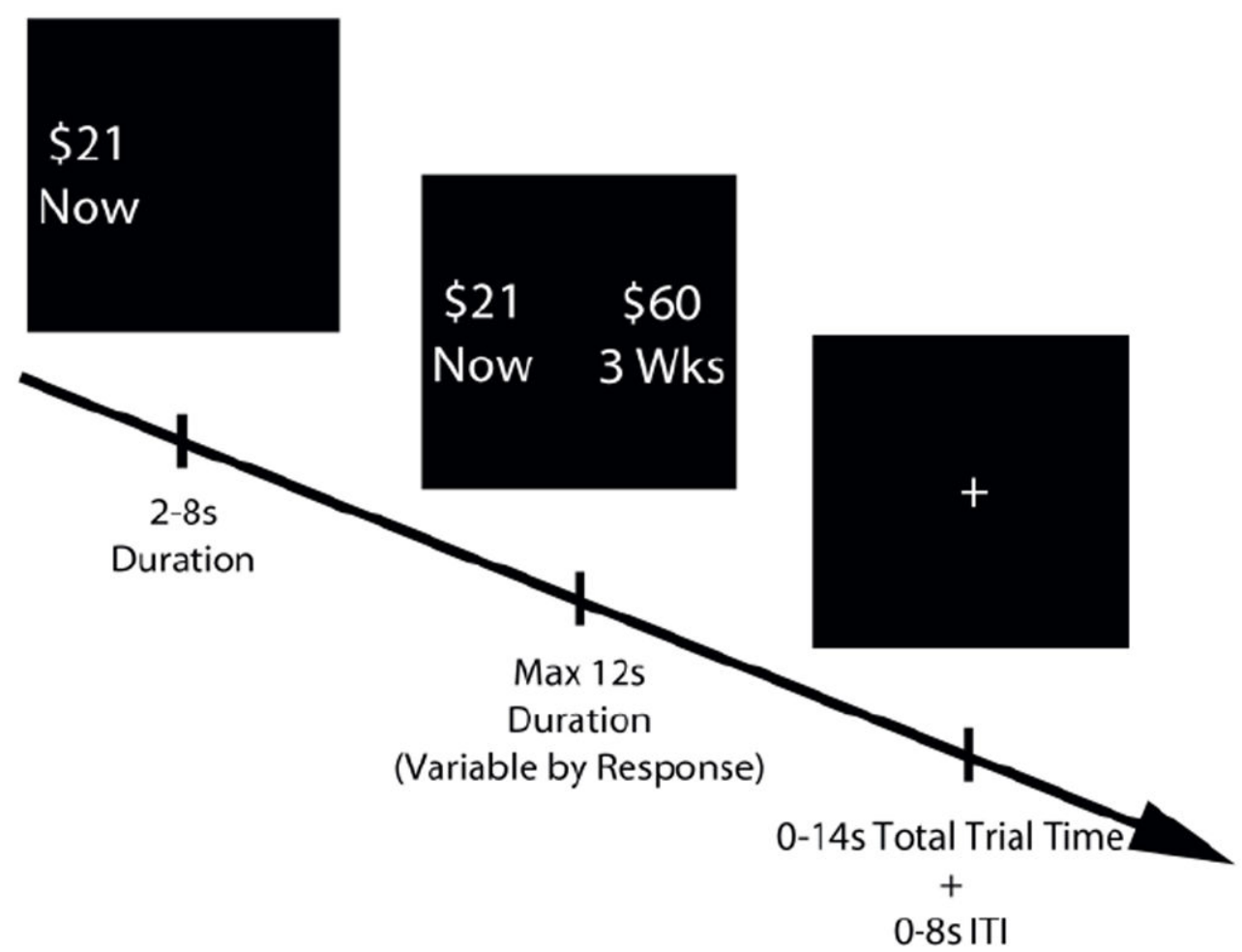

Figure 1.

An example trial, showing timing and presentation of options. Each option consisted of a dollar amount paired with a delay. The first option was displayed (side of presentation counterbalanced) for 2-8s, followed by the appearance of a second option for 6-12s (14s first option duration) or until a response was made. A fixation cross was presented for any remainder of the $14 \mathrm{~s}$ trial period, and for a $0-8 \mathrm{~s}$ inter-trial interval. 

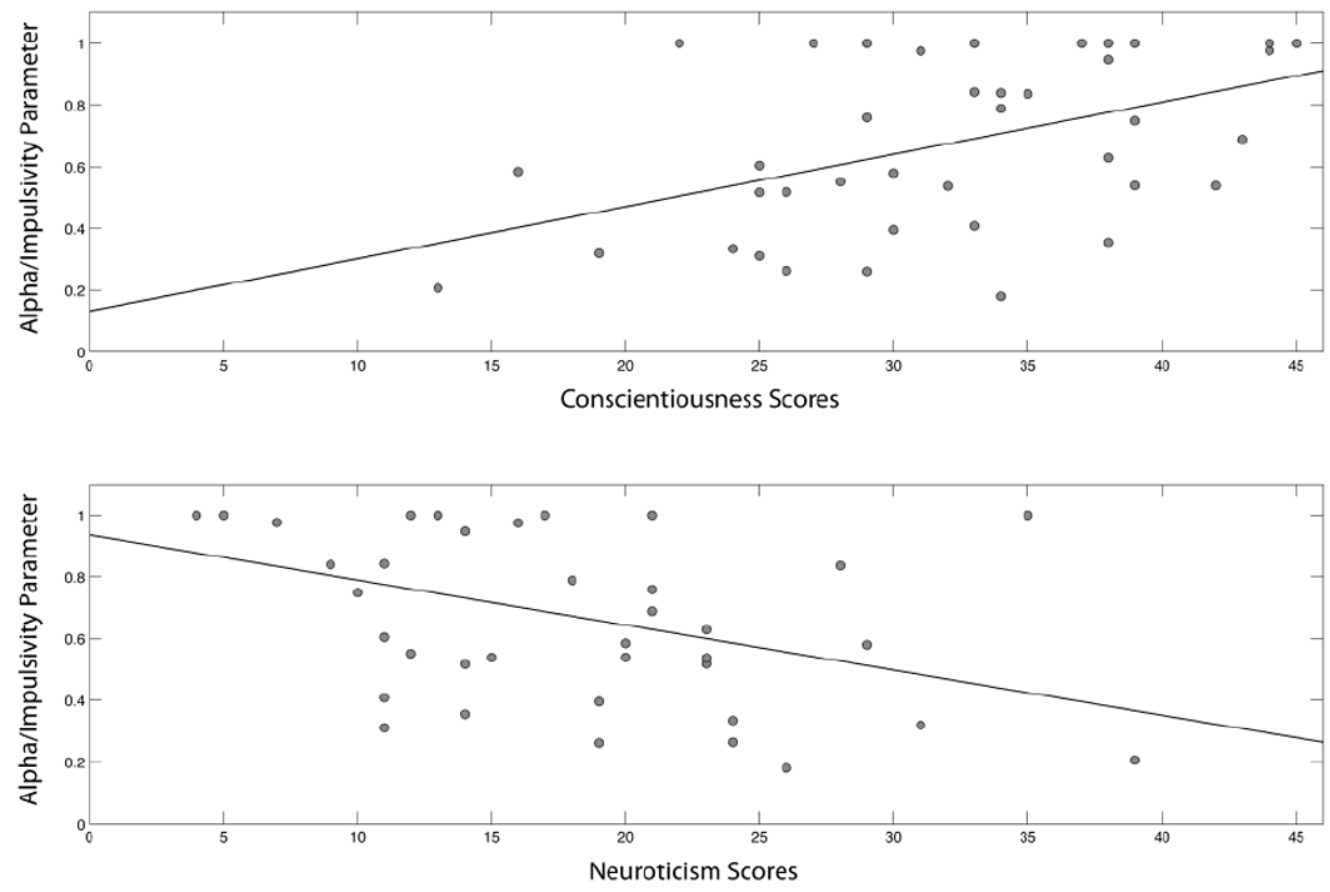

Figure 2.

Scatter plot for each individual's conscientiousness score and impulsivity parameter $a(r=$. $47, p=.002$, top), and for each individual's neuroticism score and impulsivity parameter $a$ $(r=-.41, p=.008$, bottom $)$. 


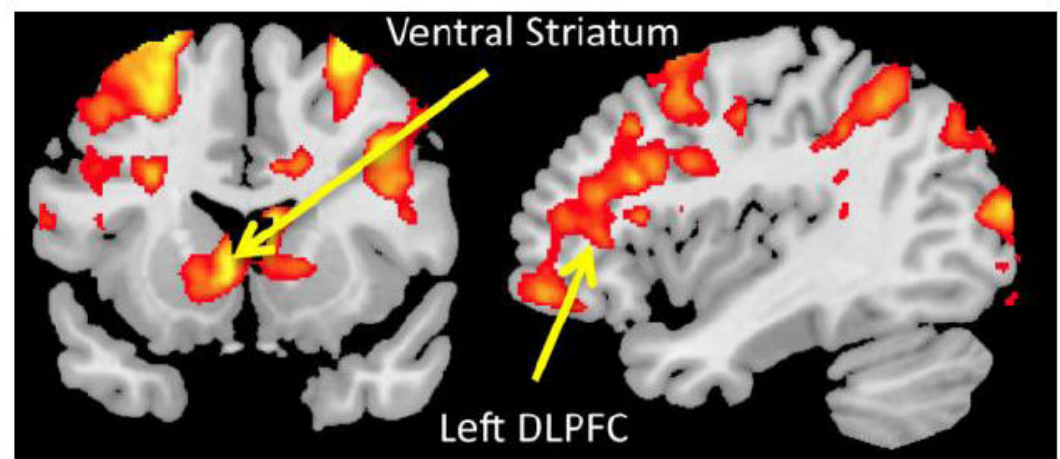

Figure 3.

Whole brain correlation of conscientiousness with the Utility Surplus Short vs. Utility Surplus Long contrast. Peak voxel of the ventral striatum: $t=3.77$, MNI coordinates: $(-6$, $15,3)$. Peak voxel of the left DLPFC/middle frontal gyrus cluster: $t=3.12$, MNI coordinates: $(-41,24,25)$ (clusterwise $\left.\mathrm{p}_{\mathrm{FDR}}<.05\right)$. 


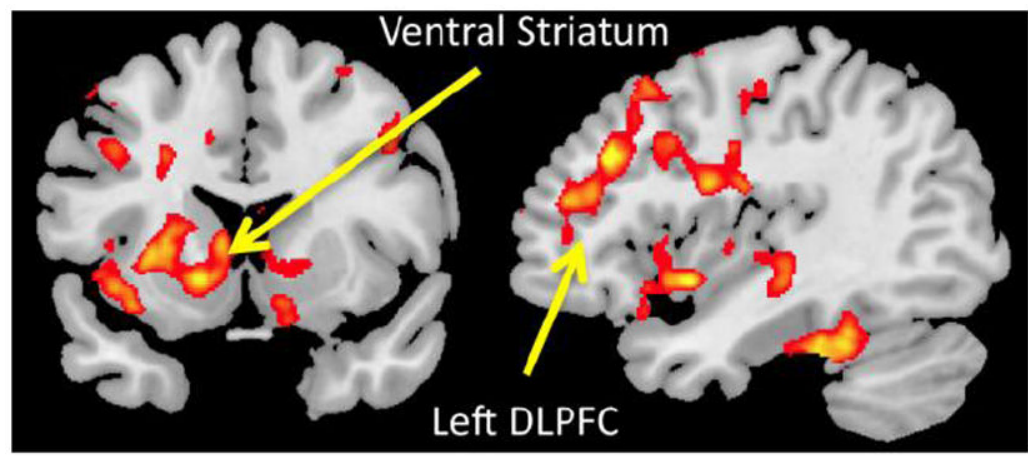

Figure 4.

Whole brain correlation of neuroticism with the Utility Surplus Long vs. Utility Surplus Short contrast. Peak voxel of the ventral striatum: $t=3.36$, MNI coordinates: $(-14,10,-6)$. Peak voxel of the left DLPFC/middle frontal gyrus cluster: $t=4.08$, MNI coordinates: $(-37$, $29,30)$ (clusterwise $\mathrm{p}_{\mathrm{FDR}}<.05$ ). 

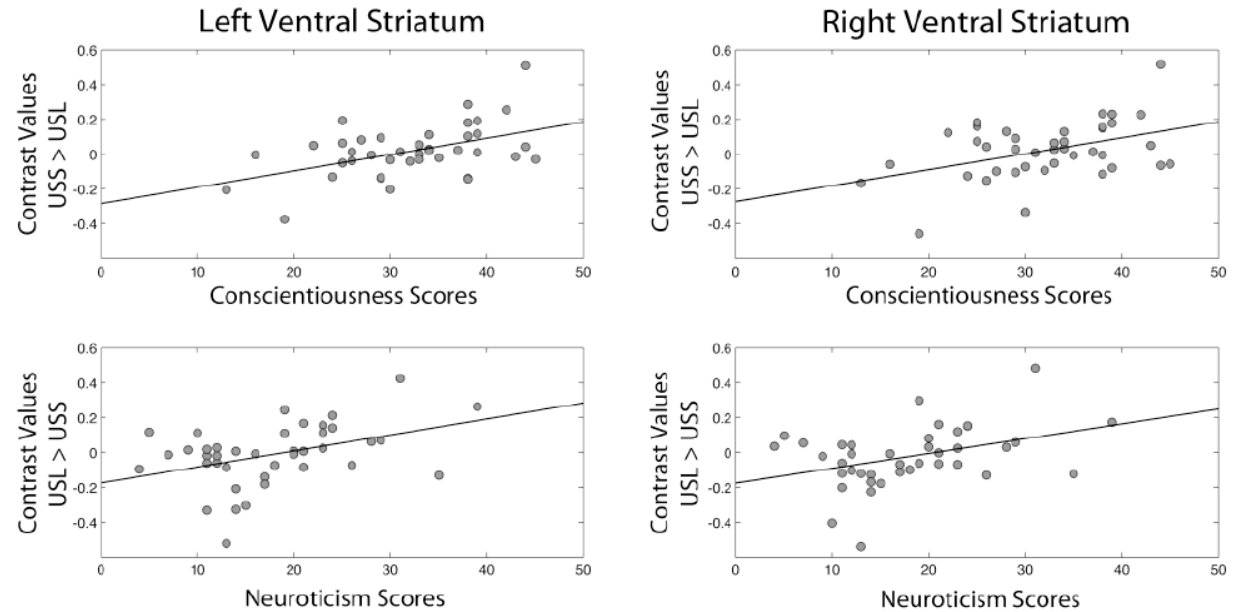

Figure 5.

Scatter plots for each individual's conscientiousness score vs. fMRI contrast parameter values for the left ventral striatum and right ventral striatum for the contrast Utility Surplus Short > Utility Surplus Long (USS > USL) (top), and for each individual's neuroticism score vs. left ventral striatum and right ventral striatum for the contrast Utility Surplus Long > Utility Surplus Short (USL > USS (bottom). Contrast values were extracted from each individual's first level contrast map. 


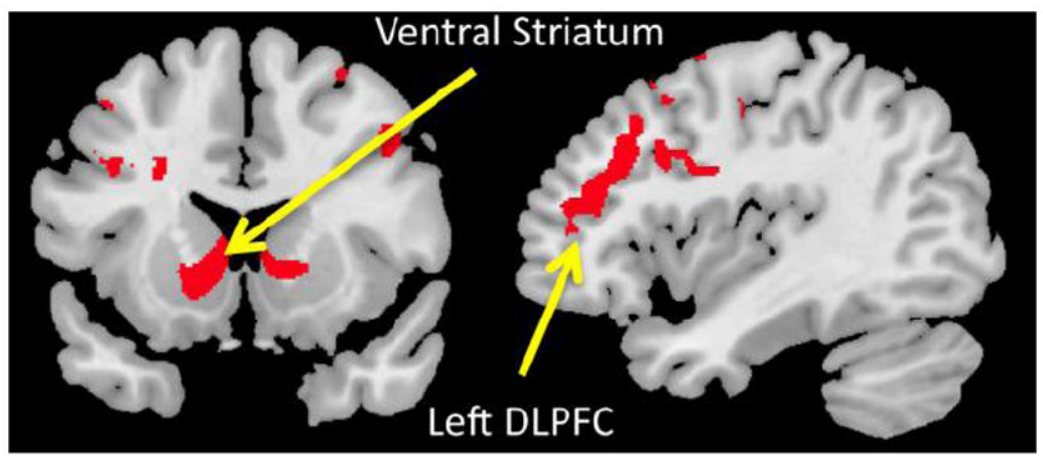

Figure 6.

Conjunction of the two thresholded (FDR < .05) correlation maps: (1) The contrast Utility Surplus Short vs. Utility Surplus Long correlated with conscientiousness (Figure 3) and (2) the contrast Utility Surplus Long vs. Utility Surplus Short correlated with neuroticism (Figure 4). 